RECEIVED

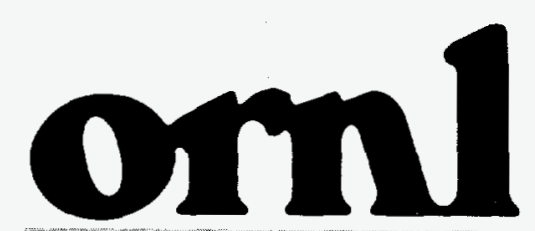

OAK RIDGE
NATIONAL
LABORATORY

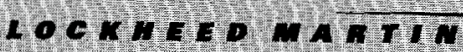
AU6 06997 OSTI

Computer Science and Mathematics Division

Distributed Intelligent Systems Section

\title{
ROBUST TRACKING WTTH SPATIO-VELOCITY SNAKES: KALMAN FILTERING APPROACH
}

\author{
Natan Peterfreund \\ Center for Engineering Systems Advanced Research (CESAR) \\ Oak Ridge National Laboratory \\ P.O. Box 2008, Bldg. 6010 \\ Oak Ridge, TN 37831-6355 \\ Email:v4p@mars.epm.ornl.gov
}

Date Published: June 1997

Research supported by the Office of Basic Energy

Sciences, U.S. Department of Energy

Prepared by the

Oak Ridge National Laboratory

Oak Ridge, Tennessee 37831

managed by

Lockheed Martin Energy Research Corporation for the

U.S. DEPARTMENT OF ENERGY

under Contract No. DE-AC05-96OR22464

\section{MASTER JWW}


This report has been reproduced directly from the best available copy.

Available to DOE and DOE contractors from the Office of Scientific and Technical Information, P. O. Box 62, Oak Ridge, TN 37831; prices available from (423) 576-8401, FTS 626-8401.

Available to the public from the National Technical Information Service, U.S. Department of Commerce, 5285 Port Royal Road, Springfield, VA 22161.

This report was prepared as an account of work sponsored by an agency of the United States Government. Neither the United States Government nor any agency thereof, nor any of their employees, makes any warranty, express or implied, or assumes any legal liability or responsibility for the accuracy, completeness, or usefulness of any information, apparatus, product, or process disclosed, or represents that its use would not infringe privately owned rights. Reference herein to any specific commercial product, process, or service by trade name, trademark, manufacturer, or otherwise, does not necessarily constitute or imply its endorsement, recommendation, or favoring by the United States Government or any agency thereof. The views and opinions of authors expressed herein do not necessarily state or reflect those of the United States Government of any agency thereof. 


\section{DISCLAIMER}

Portions of this document may be illegible electronic image products. Images are produced from the best available original document. 
ORNL/TM-13449

Computer Science and Mathematics Division

Distributed Intelligent Systems Section

\title{
ROBUST TRACKING WITH SPATIO-VELOCITY SNAKES: KALMAN FILTERING APPROACH
}

\author{
Natan Peterfreund \\ Center for Engineering Systems Advanced Research (CESAR) \\ Oak Ridge National Laboratory \\ P.O. Box 2008, Bldg. 6010 \\ Oak Ridge, TN 37831-6355 \\ Email:v4p@mars.epm.ornl.gov
}

Date Published: June 1997

Research supported by the Office of Basic Energy

Sciences, U.S. Department of Energy

Prepared by the

Oak Ridge National Laboratory

Oak Ridge, Tennessee 37831

managed by

Lockheed Martin Energy Research Corporation

for the

U.S. DEPARTMENT OF ENERGY

under Contract No. DE-AC05-96OR22464 

. . 


\section{Contents}

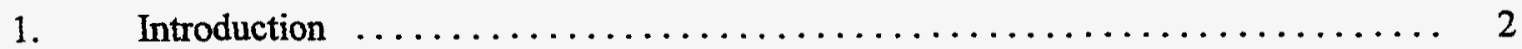

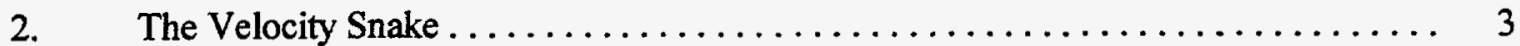

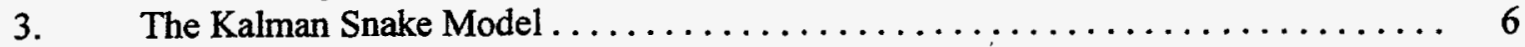

$3.1 \quad$ Real Time Mode ............................... 6

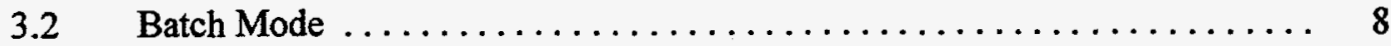

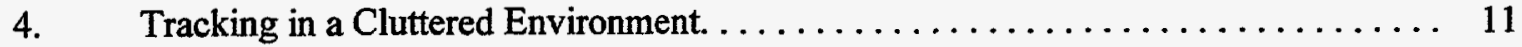

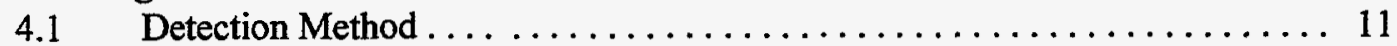

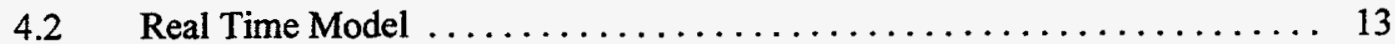

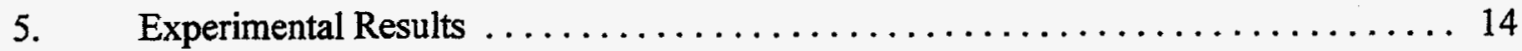

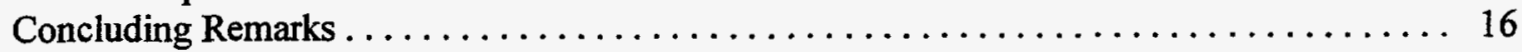

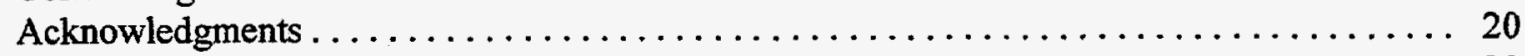

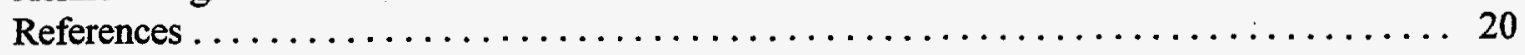





\title{
Robust Tracking with Spatio-Velocity Snakes: Kalman Filtering Approach
}

\author{
Natan Peterfreund \\ Center for Engineering Systems Advanced Research (CESAR) \\ Oak Ridge National Laboratory, P.O.Box 2008 \\ Oak Ridge TN 37831-6355. email: v4p@mars.epm.ornl.gov
}

\begin{abstract}
Using results from robust Kalman filtering, we present a new Kalman filter-based snake model for tracking of nonrigid objects in combined spatio-velocity space. The proposed model is the stochastic version of the velocity snake, an active contour model for combined tracking of position and velocity of nonrigid boundaries. The proposed model uses image gradient and optical flow measurements along the contour as system measurements. An optical-flow based measurement error is used to detect and reject image measurements which correspond to image clutter or to other objects. The method was applied to object tracking of both rigid and nonrigid objects, resulting in good tracking results and robustness to image clutter, occlusions and numerical noise.
\end{abstract}




\section{Introduction}

Considerable work has been done during the past few years in boundary tracking and motion analysis of nonrigid objects [2], [19], [11] and [10]. When vision systems are employed to track moving objects, a smooth motion of the object manifests as a nonrigid motion of the perspective image boundary. This property holds both for rigid and nonrigid objects. A common technique for visual tracking of nonrigid boundaries is the snake model: a deformable contour which moves under the influence of image forces [19]. This technique is mainly used for boundary detection in cluttered environments. Recently a new snake model was proposed, the velocity snake, an active contour for real time tracking in combined spatiovelocity space [15], [16]. The proposed work presents a robust kalman filtering approach for this model.

Boundary tracking based on deformable planar contours, known as snakes, was originally introduced by Terzopoulos et. al. (e.g. [19], [10]). Snakes are deformable contours that move under the influence of image-gradient forces, subject to certain internal deformation constraints. The contour dynamics are given by the Euler-Lagrange equations of motion associated with the contour potential. In boundary tracking problems this potential relates to the gradient of image-intensity. Considerable work has been done to overcome the numerical problems associated with the solution of the equations of motion and to improve robustness to image clutter and occlusions. This includes curve evolution methods [13] and model-based boundary representation [6], [7] [14] and [18]. Recently a new snake model was proposed for real-time tracking in combined spatio-velocity space [15], [16]. Unlike existing methods, this model uses optical-flow based velocity measurements in excess to image-gradient measurements. Treating the image sequence as continuous measurements along time, it was shown that the proposed model converges to a boundary moving at constant velocity. This is in contrast to the original snake model which was proven to be biased due to image velocity [15].

Both the velocity snake model and the original one, however, do not have an evaluation 
mechanism to detect and reject spurious measurements. These may appear in the presence of image clutter, occlusions or image noise. These measurements could cause for "cracks" in the tracking contour where parts of the contour converge to other objects in the scene. A mechanism for detection and evaluation of measurement importance within a probabilistic estimation framework, is the Kalman Filtering method. Under white Gaussian assumption of measurements noise, this method provides the optimal Bayes estimator [5] of state in a linear dynamical system. The result is given in terms of state expectation and covariance. Under the Gaussian assumption, this sufficient statistics can be used to detect and reject measurements which violate the Gaussian assumption [5]. Applications of this method can be found in target/multitarget point tracking [5], image motion tracking [4], and robust appearance-based recognition [17]. Kalman filtering versions of active contours can be found in [19] and [6]. These models, however, do not use image velocity measurements and do not have the mechanism to detect and reject spurious edges. In the following we present a robust Kalman filtering approach for the velocity snake proposed in [15] and [16]. As will be shown, this model has a natural mechanism to reject measurements which belong to other objects or to image clutter.

\section{The Velocity Snake}

In this section we present the basics of the Velocity Snake proposed in [15] and [16]. This model results from the least squares solution to boundary-contour tracking in combined spatio-velocity space.

Consider the closed contour $v(s, t)=(x(s, t), y(s, t))$ for some spatial parametric domain

$s \in[0,1]$ and time $t \in[0, \infty)$. Let $v_{s} \triangleq \frac{\partial v}{\partial s}$ and $v_{s s} \triangleq \frac{\partial^{2} v}{\partial s^{2}}$. The Lagrangian energy of the snake, originally proposed by Terzoupulos et. al. [19], [15], is given by

$$
\frac{1}{2} \int_{0}^{1}\left(w_{1}\left\|v_{s}\right\|^{2}+w_{2}\left\|v_{s s}\right\|^{2}\right) d s+\frac{1}{2} \int_{0}^{1} \mu\left\|v_{t}\right\|^{2} d s+\int_{0}^{1} P(v, t) d s
$$

The first term in this equation defines the internal deformation energy with $w_{1}(s)$ and $w_{2}(s)$ 
controlling tension and rigidity, respectively. The second and third terms are the kinetic and potential field energies of the contour. Given the image sample at time $t, I(\mathbf{x}, t)$, where $\mathbf{x}=(x, y)$ denote the spatial coordinates, typical potential field energies are functions of image intensity with $P= \pm I(\mathrm{x}, t)$, or contrast, with $P= \pm\|\nabla I(\mathrm{x}, t)\|$, where $\nabla I=$ $\left(I_{x}, I_{y}\right)$ denotes the spatial gradient of $I(\mathbf{x}, \cdot)$. Let $v^{b}$ and $v_{t}^{b}$ denote the boundary position and velocity, respectively. The energy dissipation function which is used to dampen the Langrangian energy (2.1) is given by [15]

$$
D\left(v_{t}, v_{t}^{b}\right)=\frac{\gamma}{2} \int_{0}^{1}\left\|L^{T}\left(v_{t}-v_{t}^{b}\right)\right\|^{2} d s+\frac{\beta}{2} \int_{0}^{1}\left\|\frac{\partial}{\partial s} v_{t}\right\|^{2} d s \quad \gamma>0, \beta>0
$$

where $L$ is a real matrix. The second term represents a smoothness constraint. Assuming the Lagrangian (2.1) and the above dissipation function, the Euler-Lagrange equations of motion of the velocity snake are then given by [15]

$$
\mu v_{t t}+\gamma C\left(v_{t}, v_{t}^{b}\right)-\beta \frac{\partial}{\partial s}\left(\frac{\partial}{\partial s} v_{t}\right)-\frac{\partial}{\partial s}\left(w_{1} v_{s}\right)+\frac{\partial^{2}}{\partial s^{2}}\left(w_{2} v_{s s}\right)=-\nabla P(v(s, t), t)
$$

where the velocity control term $C\left(v_{t}, v_{t}^{b}\right)$ satisfies

$$
C\left(v_{t}, v_{t}^{b}\right)=L L^{T}\left(v_{t}-v_{t}^{b}\right)
$$

Since the velocity of the object's boundary is unknown in advance, we use instead the apparent velocity $v_{t}^{i}$ of the image at the contour position. In [15] and [16] we showed that the contour will converge to the boundary moving at constant velocity if the initial contour is sufficiently close to the boundary of the moving object, and if we have the velocity of the object at the contour position (which could be computed via optical flow techniques). On the other hand, we showed that the lack of motion control in the original model, proposed in [19], causes a bias in the contour position. This could lead to serious tracking problems even in the case of non cluttered environments [15].

Various techniques for estimating the apparent velocity $v_{t}^{i}$ of the the image at the contour position had been proposed in [15] and [16]. These methods are based on the optical flow constraint equation [9]

$$
\nabla I \cdot v_{t}^{i}+I_{t}=0
$$


and on integration of measurements along time [3].

A special case of interest of (2.3), with $v_{t}^{b}$ replaced by the apparent motion $v_{t}^{i}$, is when $L=\nabla I(v(s, t))$. In this case the snake model $(2.3)$ degenerates to the optical flow constraint model [15]

$$
\mu v_{t t}+\gamma \nabla I\left(\nabla I \cdot v_{t}+I_{t}\right)-\beta \frac{\partial}{\partial s}\left(\frac{\partial}{\partial s} v_{t}\right)-\frac{\partial}{\partial s}\left(w_{1} v_{s}\right)+\frac{\partial^{2}}{\partial s^{2}}\left(w_{2} v_{s s}\right)=-\nabla P(v(s, t), t) .
$$

This result is due to the constraint equation (2.5). In this model there is no need to estimate the image velocity because the optical flow term provides a measure of the error in velocity estimation. However, this scheme is more sensitive to measurements and numerical noise than the model given in (2.3), as instead of the velocity error, we have a projected version on the direction of $\nabla I$.

Next we consider the discretization of the velocity snake model in space. Consider equidistant sampling of the contour $v(s, \cdot)$ along $s$ with a sampling distance $h(h>0)$, and let $u=\left[u_{1}, \ldots, u_{M}\right]$ be the vector of samples with $u_{i}=v\left(s_{i}, \cdot\right)$ denoting the $\mathrm{i}$-th sample of $v(s, \cdot)$. Using finite difference approximation,

$$
v_{s}\left(s_{i}\right) \approx\left(u_{i+1}-u_{i}\right) / h \text { and } v_{s s}\left(s_{i}\right) \approx\left(u_{i+1}-2 u_{i}+u_{i-1}\right) / h^{2},
$$

the discrete versions of (2.3) and (2.6) are then given by

$$
\mu u_{t t}+\gamma C\left(u_{t}, u_{t}^{i}\right)+\beta D D^{T} u_{t}+K u=-\nabla P(u, t)
$$

where for (2.3) we have

$$
C\left(u_{t}, u_{t}^{i}\right)=L L^{T}\left(u_{t}-u_{t}^{i}\right)
$$

and for $(2.6)$ we have

$$
C\left(u_{t}, u_{t}^{i}\right)=\nabla I\left(\nabla I \cdot u_{t}+I_{t}\right) .
$$

The matrix $K$ consists of the deformation constraints imposed by $w_{1}$ and $w_{2}$ in (2.3) [19], and $D$, the derivative matrix, is defined by

$$
D_{i j}=\left\{\begin{array}{rl}
-1 & \text { if } \mathrm{i}=\mathrm{j} \\
1 & \text { if } \mathrm{j}=\mathrm{i}+1 \\
0 & \text { otherwise }
\end{array} .\right.
$$




\section{The Kalman Snake Model}

Tending to minimize the difference between the contour and boundary position and velocity, the proposed model (2.3) provides deterministic estimation of boundary position and velocity. Alternatively, this problem could be treated as random parameter estimation. Under the assumptions of Gaussian prior probabilities of state and measurements noise, the velocity and state parameters of the tracking contour could be obtained by the Kalman Filtering method (e.g. [8]). In the following we propose a Kalman filtering approach for state estimation of the tracking contour. Unlike the deterministic, least-squares based, approach proposed in [15], this method allows systematic integration of measurements along time and the rejection of spurious measurements.

Like in the deterministic model we propose two modes of estimation [15]: Batch Mode, in which the velocity is estimated independently of the contour dynamics, and treated as an input to the tracking model, and Real Time Mode, which uses the velocity measurements to adjust the probability distribution function of the contour parameters.

\subsection{Real Time Mode}

Consider the discrete model (2.8) with $\gamma=0$. Let $\xi=\left[x_{1}, x_{2} \ldots x_{M}\right]^{T}$ and $\eta=\left[y_{1}, y_{2} \ldots y_{M}\right]^{T}$ denote the sampling vectors corresponding to $u$, with $u_{i}=\left[x_{i}, y_{i}\right], V=\left[\xi^{T}, \eta^{T}\right]^{T}$ and $\dot{V}=$ $\left[\dot{\xi}^{T}, \dot{\eta}^{T}\right]^{T}$, where $\dot{V}$ denote the derivative of $V$ with respect to $t$. We denote the Gaussian probability density with mean vector $h$ and covariance matrix $Q$ by $N(h, Q)$.

The Markov process model corresponding to $(2.8)(\gamma=0)$ is given by

$$
\frac{d}{d t}\left[\begin{array}{c}
V \\
\dot{V}
\end{array}\right]=\left[\begin{array}{cc}
O_{2 M \times 2 M} & I_{2 M} \\
-\frac{1}{\mu} \Sigma & -\frac{\beta}{\mu} \Psi \Psi^{T}
\end{array}\right]\left[\begin{array}{c}
V \\
\dot{V}
\end{array}\right]-\frac{1}{\mu}\left[\begin{array}{c}
O_{2 M \times 2 M} \\
I_{2 M}
\end{array}\right] u^{p}+\left[\begin{array}{c}
0_{2 M} \\
q
\end{array}\right] q \sim N(0, Q)
$$

where $O_{N \times M}$ is an $N \times M$ zero matrix, $I_{2 M}$ is a $2 M \times 2 M$ Identity matrix, $0_{2 M}$ is $2 M$ 
dimensional vector of zeroes,

$$
\Sigma=\left[\begin{array}{cc}
K & O_{M \times M} \\
O_{M \times M} & K
\end{array}\right] \text { and } \Psi=\left[\begin{array}{cc}
D & O_{M \times M} \\
O_{M \times M} & D
\end{array}\right]
$$

Let $\Pi=\left[\Pi_{x}, \Pi_{y}\right]$, where $\Pi_{x}$ and $\Pi_{y}$ are diagonal matrices with the diagonal elements given by $I_{x}(u)$ and $I_{y}(u)$, respectively, and $\partial P=\left[P_{x}^{T}(u), P_{y}^{T}(u)\right]^{T}$. The measurements vector, composed of $\partial P(V, t)$ and the optical-flow constraint equation (2.5), is given by

$$
z=\left[\begin{array}{c}
u^{p} \\
I_{t}
\end{array}\right]=\left[\begin{array}{c}
\partial P(V, t) \\
-\Pi \dot{V}
\end{array}\right]+\left[\begin{array}{c}
0_{2 M} \\
w
\end{array}\right] w \sim N(0, W)
$$

Note that the second measurement vector $I_{t}$ results from the first order approximation of the intensity preserving equation

$$
I(V, t+d t)=I(V-\dot{V} d t, t) .
$$

One of the major problems in gradient-based boundary tracking, is the appearance of spurious edges which could "trap" parts of the tracking contour. Within the proposed measurements model (3.2), however, edges whose velocity measurements were found to be inconsistent with previous velocity estimation, are considered to be spurious. This allows for systematic filtering of components in $\nabla P(V, t)$ which do not correspond to the boundary being tracked.

A method for solving the state estimation of (3.1), subject to measurement vectors of the form (3.2), is the Extended Kalman Filter (e.g. [8]). This method uses linearization techniques to obtain simple approximate solution, similar to the one obtained for linear systems, to nonlinear estimation problems.

Using the Extended Kalman Filter, the state estimation of (3.1) satisfies

$$
\frac{d}{d t}\left[\begin{array}{c}
\hat{V} \\
\hat{V}
\end{array}\right]=\left[\begin{array}{cc}
O_{2 M \times 2 M} & I_{2 M} \\
-\frac{1}{\mu} \Sigma & -\frac{\beta}{\mu} \Psi \Psi^{T}
\end{array}\right]\left[\begin{array}{c}
\hat{V} \\
\hat{\dot{V}}
\end{array}\right]-\frac{1}{\mu}\left[\begin{array}{c}
O_{2 M \times 2 M} \\
I_{2 M}
\end{array}\right] \partial P(\hat{V}, t)+K(\hat{V}, t)\left(I_{t}(\hat{V}, t)+\Pi \dot{V}\right)
$$


where, for

$$
H=\left[O_{M \times 2 M},-\Pi\right] \text { and } L=\left[\begin{array}{cc}
L_{1} & L_{12} \\
L_{12}^{T} & L_{2}
\end{array}\right]
$$

we have the Kalman gain matrix

$$
K(\hat{V}, t)=L H^{T} W^{-1}=-\left[\begin{array}{c}
L_{12} \\
L_{2}
\end{array}\right] \Pi^{T} W^{-1}
$$

The covariance matrix $L$ of the state estimation is given by the solution of the matrix Riccati equation (e.g. [8])

$$
\dot{L}=F L+L F-L H^{T} W^{-1} H L+\bar{Q}
$$

where, for $\partial^{2} P_{i j}=\frac{\partial}{\partial v_{j}} \partial P_{i}$, we have

$$
F=\left[\begin{array}{cc}
O_{2 M \times 2 M} & I_{2 M} \\
-\frac{1}{\mu}\left(\Sigma+\partial^{2} P\right) & -\frac{\beta}{\mu} \Psi \Psi^{T}
\end{array}\right] \text { and } \bar{Q}=\left[\begin{array}{cc}
O_{2 M \times 2 M} & O_{2 M \times 2 M} \\
O_{2 M \times 2 M} & Q
\end{array}\right]
$$

In the special case where $L_{12}=O_{2 M \times 2 M}, L_{2}=I_{2 M}$ and $W=\frac{1}{\gamma} I_{M}$, the random estimation (3.4) degenerates to the deterministic model defined by (2.8) and (2.10). The latter, however, lacks the qualitative means according to which the importance of a given measurement is evaluated and incorporated within the estimation process.

\subsection{Batch Mode}

In this section we consider the random estimation approach to the velocity snake model (2.3). The apparent velocity of the image at the contour is assumed to be a first-order Markov process.

The first order form of the velocity snake model (2.8), with the velocity control (2.9) and $L=I_{2 M}$, is given by

$\frac{d}{d t}\left[\begin{array}{c}V \\ \dot{V}\end{array}\right]=\left[\begin{array}{cc}O_{2 M \times 2 M} & I_{2 M} \\ -\frac{1}{\mu} \Sigma & -\frac{1}{\mu}\left(\beta \Psi \Psi^{T}+\gamma I\right)\end{array}\right]\left[\begin{array}{c}V \\ \dot{V}\end{array}\right]-\frac{1}{\mu}\left[\begin{array}{c}O_{2 M \times 2 M} \\ I_{2 M}\end{array}\right] u^{p}+\frac{\gamma}{\mu}\left[\begin{array}{c}O_{2 M \times 2 M} \\ I_{2 M}\end{array}\right] U_{t}^{i}$, 
where the apparent velocity vector $U_{t}^{i}=\left[\mathbf{x}_{t}^{i}, \mathbf{y}_{t}^{i}\right]$ and $\mathbf{x}_{t}^{i}$ and $\mathbf{y}_{t}^{i}$ are the components of the apparent velocity on $u$ in the $x$ and $y$ directions, respectively. We assume that the apparent velocity of the image at the contour obeys the differential equation

$$
\frac{d}{d t} U_{t}^{i}=-\alpha U_{t}^{i}+q \quad \alpha>0, \quad q \sim N(0, Q) .
$$

The measurement vector corresponding to image potential and optical-flow constraint equation, is given by

$$
\begin{aligned}
& u^{p}=\partial P \\
& z=\left[\begin{array}{c}
I_{t} \\
0
\end{array}\right]=\left[\begin{array}{c}
-\Pi \\
\Psi
\end{array}\right] U_{t}^{i}+\left[\begin{array}{l}
w_{1} \\
w_{2}
\end{array}\right] \quad w_{i} \sim N\left(0, W_{i}\right) .
\end{aligned}
$$

The first component in $z$ correspond to the optical flow constraint equation (2.5). The second term measures the smoothness of the apparent velocity along the contour line. The same modeling of the measurement vector $z$, in the context of optical flow estimation, can be found in [12]. Using the Kalman Filtering equations (e.g. [8]), the estimate of the apparent velocity satisfies

$$
\left.\frac{d}{d t} \hat{U}_{t}^{i}=-\alpha \hat{U}_{t}^{i}+L\left(-\Pi^{T} W_{1}^{-1}\left(I_{t}+\Pi \hat{U}_{t}^{i}\right)-\Psi^{T} W_{2}^{-1} \Psi U_{t}^{i}\right)\right)
$$

where, by (3.7), the covariance matrix $L$ is given by the solution of the matrix Riccati equation

$$
\dot{L}=-2 \alpha L-L\left(\Pi^{T} W_{1}^{-1} \Pi+\Psi^{T} W_{2}^{-1} \Psi\right) L+Q .
$$

The solution $\hat{U}_{t}^{i}$ of (3.12) and $u^{p}=\partial P$ are used as control inputs to the velocity snake model (3.9). As in the real time mode, measurements equations whose error exceeds certain threshold and the corresponding components in $\partial P$, are considered to be spurious and thus being ignored.

Next we present a structure based estimation approach for image velocity. Under the assumption of tracking a given 3-D model, this approach allows for space dependent integration of measurements along the contour, resulting in low variance velocity estimation. Within the proposed model, however, we limit the application only to shallow objects. 
Let $(X, Y, Z)$ represent the Cartesian coordinate system of the camera and let $(x, y)$ represent the corresponding coordinates of the image plane, with the $x$ and the $y$ axes parallel to $X$ and $Y$, respectively. The origin of the planar image is given by $(X, Y, Z)=(0,0,1)$. Relative to the camera coordinate system $(X, Y, Z)$, it was shown that the projected velocity $\mathbf{w}=\left[w_{x}, w_{y}\right]^{T}$ of a point undergoing translation with a translation velocity $T=\left(T_{X}, T_{Y}, T_{Z}\right)$ and rotation with a rotation velocity $\Omega=\left(\Omega_{X}, \Omega_{Y}, \Omega_{Z}\right)$, satisfies (e.g. [1])

$$
\begin{aligned}
& w_{x}=-\Omega_{X} x y+\Omega_{Y}\left(1+x^{2}\right)-\Omega_{Z} y+\left(T_{X}-T_{Z} x\right) / Z \\
& w_{y}=-\Omega_{X}\left(1+y^{2}\right)+\Omega_{Y} x y+\Omega_{Z} x+\left(T_{Y}-T_{Z} y\right) / Z
\end{aligned}
$$

According to the above result the image velocity is linear in the velocity components. This property can allow for an analytic solution to the velocity estimation problem, if the depth term $Z$ in each of the image points is known. Under the assumption that the imaged region is a planar surface, i.e., $k_{X} X+k_{Y} Y+k_{Z} Z=1$ for some real scalars $k_{X}, k_{Y}$ and $k_{Z}$, the image velocity (3.14) becomes the following transformation [1]

$$
\begin{aligned}
& w_{x}=a_{1}+a_{2} x+a_{3} y+a_{7} x^{2}+a_{8} x y \\
& w_{y}=a_{4}+a_{5} x+a_{6} y+a_{7} x y+a_{8} y^{2}
\end{aligned}
$$

for some scalars $a_{1}, \ldots, a_{8}$. Let $\psi_{u_{t}^{i}}=\left[a_{1} \ldots a_{8}\right]^{T}$. Similar to image velocity model (3.10) we define the velocity parameters model as

$$
\frac{d}{d t} \psi_{u_{t}^{i}}=-\alpha \psi_{u_{t}^{i}}+r \quad \alpha>0, \quad r \sim N(0, R)
$$

Normally, the covariance matrix $R$ is considered to be diagonal. The measurement vector corresponding to (3.11) is then given by

$$
\begin{aligned}
& u^{p}=\partial P \\
& z=I_{t}=H \psi_{u_{i}^{i}}+w \quad w \sim N(0, W)
\end{aligned}
$$

where

$$
H=-\Pi\left[\begin{array}{cccccccc}
1_{M} & \xi & \eta & 0_{M} & 0_{M} & 0_{M} & \xi^{2} & \xi \cdot \eta \\
0_{M} & 0_{M} & 0_{M} & 1_{M} & \xi & \eta & \xi \cdot \eta & \eta^{2}
\end{array}\right]
$$


$1_{M}$ is an $M$ dimensional vector of ones, $\xi_{i} \xi_{i} \triangleq\left[\xi^{2}\right]_{i}$ and $\xi_{i} \eta_{i} \triangleq[\xi \cdot \eta]_{i}$. In this model we do not have a measure of velocity smoothness since the velocity model is already smooth. The state estimation of (3.16) (and (3.9)) is performed by the Kalman Filtering method, similar to state estimation in the original model (3.9) and (3.10).

\section{Tracking in a Cluttered Environment}

The random estimation approach proposed in previous subsections provides a probabilistic method for detecting spurious measurements, i.e. measurements which avoids the Gaussian assumption of noise or belong to other targets.

A measurement is treated as noise or spurious if the error in the measurement equation exceeds certain threshold. Usually this threshold corresponds to low probability of measurements values. In the following we use this method to detect snake points with spurious measurements of velocity and potential fields. Within the proposed framework, snake points $u_{i}=\left(x_{i}, y_{i}\right)$ whose velocities measurements equations exceeds certain threshold, are discarded. The corresponding components in $u^{p}$ are replaced by zeros, the optimal estimation under zero mean assumption of $\partial P$.

\subsection{Detection Method}

Consider the Kalman Filtering problem of state estimation. Let $x$ be the system's state, and

$$
z=H x+r, \quad r \sim N(0, R)
$$

the measurement equation, for some measurement matrix $H$ and noise $r$. Prior to update of state estimation, let $\hat{x}^{-}$and

$$
\hat{z}^{-}=H \hat{x}^{-}
$$


denote the predictions of state and measurements vectors, respectively. The coveriance matrix of $\hat{z}^{-}$is given by (e.g. [5])

$$
S=H L^{-} H^{T}+R
$$

where $L^{-}$is the covariance matrix of $\hat{x}^{-}$.

Let

$$
z_{i}=H_{i} x+r_{i} \quad i=1 \ldots e
$$

with $\{z\}=\cup_{i=1}^{e}\left\{z_{i}\right\}$ denote the measurements in $z$ with uncorrelated noise, i.e., $E\left(r_{i} r_{j}^{T}\right)=$ 0 , where $r_{i}$ and $H_{i}$ are the appropriate components in the measurement noise $r$ and the measurement matrix $H$, respectively. It can be shown that serial updates of state estimation using $z_{i}$ for $i=1 \ldots e$, is equivalent to single update based on $z$ (e.g. [8]). This approach allows for independent evaluation of each of the velocity measurements, resulting in a simple rejection scheme for spurious measurements.

The detection/rejection scheme of $z_{i}$ is based on probability evaluation of having $z_{i}$, assuming it is the realization of the predicted measurement $\hat{z}_{i}^{-}=H_{i} \hat{x}^{-}$. A measurement is rejected if this probability is less then a given detection threshold (e.g. [5]). The covariance matrix of $\hat{z}_{i}^{-}$is given by

$$
S_{i}=H_{i} L^{-} H_{i}^{T}+R_{i}
$$

where $R_{i}=E\left(r_{i} r_{i}^{T}\right)$. Assuming each of the new measurements $z_{i}$, conditioned upon previous measurements vector $Z$, is normally distributed, we have

$$
P\left(z_{i} \mid Z\right)=N\left(\hat{z}_{i}^{-}, S_{i}\right) \quad i=1 \ldots e
$$

The validation region $\Omega_{i}$ of each measurement $z_{i}$ is defined as (e.g. [5])

$$
\Omega_{i}\left(\gamma_{i}\right) \triangleq\left\{z_{i}:\left[z_{i}-\hat{z}_{i}^{-}\right]^{T} S_{i}^{-1}\left[z_{i}-\hat{z}_{i}^{-}\right] \leq \gamma_{i}\right\}
$$

The value of $\gamma_{i}$ relates to probability of detection $P_{D}^{i}$ according to

$$
P_{D}^{i}=P\left(z_{i} \in \Omega_{i}\left(\gamma_{i}\right) \mid Z\right)
$$


Within the proposed detection approach, a measurement $z_{i}$ is discarded if it doesn't belong to the validation region $\Omega_{i}$. In the following we present the detection method for the Real Time model. As will be shown, the prediction of state and measurements at the $i$ 'th point of the snake, depends only on the snake dynamics and on the values of measurements at this point. No other measurements affects these predictions. This will allow for independent detection and evaluation of importance for each of the snake points. The method for detecting spurious measurements in the Batch Mode model follows directly from the above scheme.

\subsection{Real Time Model}

Consider the system model (3.1) and (3.2). We assume that the elements of $I_{t}=\left[I_{t 1} \ldots I_{t M}\right]$ are uncorrelated, i.e., $W=\sigma I_{M}$ for some $\sigma>0$, so that the validation of each measurement $I_{t i}$ could be done separately.

Let $\mathbf{V}=[V, \dot{V}]$ denote the system state, $\hat{\mathbf{V}}=[\hat{V}, \hat{\dot{V}}]$ the estimated state, and $L$ the corresponding covariance matrix. The measurements with uncorrelated noise components, corresponding to (4.4), are given by

$$
z_{i}=I_{t i}=-\Pi_{i} \dot{V}+w_{i} \quad w_{i} \sim N\left(0, \sigma^{2}\right) \quad i=1 \ldots M
$$

where $\Pi_{i}$ is the $i^{\prime}$ th row of $\Pi$. The discrete approximation of the state prediction $\hat{\mathbf{V}}^{-}$and the corresponding covariance matrix $L^{-}$are, by (3.1),

$$
\begin{aligned}
& \hat{\mathbf{V}}^{-}=\left(I_{4 M}+\Delta t\left[\begin{array}{cc}
O_{2 M \times 2 M} & I_{2 M} \\
-\frac{1}{\mu} \Sigma & -\frac{\beta}{\mu} \Psi \Psi^{T}
\end{array}\right]\right) \hat{\mathbf{V}}-\frac{\Delta t}{\mu}\left[\begin{array}{c}
O_{2 M \times 2 M} \\
I_{2 M}
\end{array}\right] u^{P} \\
& L^{-}=\left(I_{4 M}+F \Delta t\right) L\left(I_{4 M}+F \Delta t\right)^{T}+\Delta t \bar{Q}
\end{aligned}
$$

where $F$ and $\bar{Q}$ are given in (3.8) and $\Delta t$ is the sampling distance. Using (4.8), the prediction of velocity measurements and the associated variances are given by

$$
\begin{aligned}
& \hat{z}_{i}^{-}=I_{t i}=-\Pi_{i} \hat{\dot{V}}^{-} \quad i=1 \ldots M \\
& S_{i}=\Pi_{i} L_{22}^{-} \Pi_{i}^{T}+\sigma^{2} .
\end{aligned}
$$


It can be shown that both $\hat{z}_{i}^{-}$and $S_{i}$ are only functions of the velocity and potential field at the $i^{\prime}$ th sample $u_{i}=\left(x_{i}, y_{i}\right)$ of the snake position. No other components corresponding to $\partial P$ and $I_{t}$ affect these equations. Using the independence property of $\hat{z}_{i}^{-}$and $S_{i}$, each measurement $z_{i}$ is assumed to have the Gaussian distribution (4.6). The rejection/detection decision of the scalar measurement $z_{i}$ is done by evaluating the Mahalanovis distance in (4.7). Since the components in $\partial P$ corresponding to $z_{i}$ use the same gradient information of image intensity, these components are ignored and replaced by zeroes if $z_{i}$ is declared as spurious. The zero value is the optimal estimator under zero mean assumption of $\partial P$.

\section{Experimental Results}

We demonstrated the performance of the proposed contour model by applying it to real image sequences with both rigid (mobile robot) and non-rigid (human body, waving hand) objects. The initial contour lines were generated manually, forming a rough polygonal approximation to the object's boundary. This task could be done automatically via ATR -based methods followed by rough boundary approximation, as the boundary need not be determined exactly. Prior to the calculation of image gradients and velocities, the image sequences were smoothed, both in space and time, by a Gaussian filter. In the proposed examples we used a fixed Gaussian filter with $\sigma=2$, both in space and time. Spatial derivatives of the images were calculated by applying a simple $3 \times 3$ Sobel operator.

The discretization in time of the differential equations was based on the implicit differencing approximation

$$
X_{n+1}=X_{n}+\dot{X}_{n+1} \Delta t
$$

where $X_{n}$ and $\dot{X}_{n}$ are the state and the time-derivative of state at time step $n$. This scheme was found to derive the most stable results. In the simulations we used the sampling interval $\Delta t=1$.

Contour parameters: We used the contour models with a spatial sampling distance $h=5$ 
(pixels) and with $\gamma=\mu=2$. The deformation parameters $w_{1}$ and $w_{2}$ were set to constant values. More complex techniques define the values of $w_{1}$ and $w_{2}$ to be nonlinear functions of the distance between nearby points [11]. In our model, however, we found the velocity control to have similar smoothness effects, making these extensions unnecessary. The potential energy function $P=-\|\nabla I(\mathbf{x})\|$ and its directional derivatives were computed using a simple $3 \times 3$ Sobel operator. These operations were made only in the vicinity of the contour.

Image sequences: The tracking schemes were demonstrated on the following image sequences:

A. Multiple Target Scene (Figure 5.1): Lab. scene with multiple moving objects including a mobile robot and a walking woman. In parts of the image sequence the woman body occludes the mobile robot. The scene is composed of 90 images sampled at video rate.

B. Office Scene (Figure 5.3): An office scene with the author waving his hand under nonuniform lighting conditions. The scene includes image clutter such as shadows, shelves, books and a computer monitor. The sequence is composed of 60 images sampled at video rate.

Tracking results: The results of tracking the mobile robot in the Multiple Target Scene are shown in Figure 1(a)-(i). These images correspond, respectively, to frame number 5, 7, $10,14,17,20,22,25$ and 50 , in the image sequence. This example demonstrate boundary tracking of a rigid object (mobile robot) under the occlusion of a walking woman. In this example we used the Batch Mode tracking model (3.9) with the structured velocity model (3.15)-(3.17). The detection threshold of spurious measurements was set to $\gamma_{i}=1$. In the following figures we used the symbol '*' to mark the points which were detected as spurious. The position of contour points in previous frame are ploted with ' $\because$. The contour position before occlusion is shown in Figure 1(a). In the presence of occlusion, e.g., figures 5.1(b)-(f), it can be seen that nearly all occluded points were detected as spurious. In figures 5.1(b)-(c) it can be seen that while some of the snake points were detected to be occluded (on the right side of the robot), the contour keeps tracking the unoccluded parts (on the left part of the robot). This can also be seen in figures 5.1(d)-(f) where the contour starts tracking the un occluded right side of the robot while the left side is detected to be occluded. In couple of 
places, however, the tracking scheme does not detect the occlusions (figures 5.1(e)-(f) on the low left part), but the system managed to converge back to the object after the occlusion ends (figures $5.1(\mathrm{~g})-(\mathrm{h})$ ).

In figures 5.2(a)-(b) we show the results of tracking the mobile robot with the Batch Mode model and detection threshold $\gamma_{i}=4$, and in 5.2(c)-(d), the results with no detection threshold. It is shown that the performance of tracking declines as the detection threshold increases. In this case, spurious measurements, which violate the Gaussian assumption, cause for bias in the estimation of position and velocity. The results of tracking with the Kalman Snake of Terzopoulos [19] are shown in figures 5.2(e)-(f). In this case, it is shown that the lack of velocity information causes the tracking contour to switch to the occluding object.

The results of tracking the waving hand with the Real Time mode model and with detection threshold $\gamma_{i}=1$, are shown in figures 5.3(a)-(e). The images correspond, respectively, to frame number $3,18,30,40$ and 50 , in the image sequence. It can be seen that most of the points detected to be spurious belong to regions with image-clutter edges. The results of tracking with the original Kalman Snake model [19] are shown in figure 5.3(f)-(g).

\section{Concluding Remarks}

Using latest results in active contour models, we have described a new Kalman filtering approach for the velocity snake: an active contour model for visual tracking in combined spatio-velocity space. The proposed model uses image gradient and optical flow measurements along the contour, as system measurements. This model, which generalizes the least squares approach proposed in the original snake model of Terzopoulos [19], and, lately, in the velocity snake proposed by the author [15], allows for systematic integration and evaluation of importance of image measurements. The stochastic means, according to which the importance of a new measurement is evaluated, allows the detection of image measurements which belong to other objects. These measurements are then ignored. As optical-flow components 


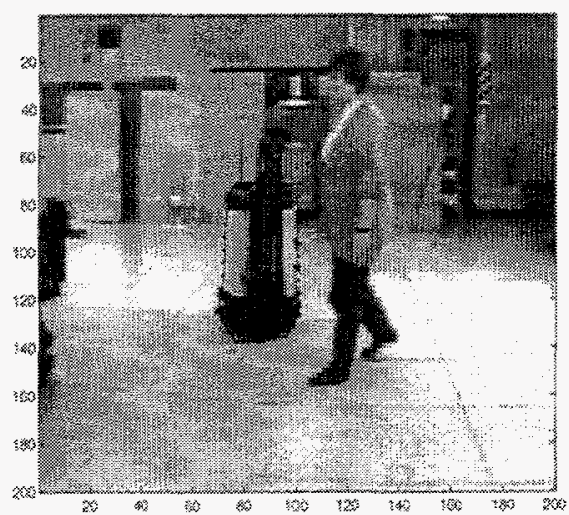

(a)

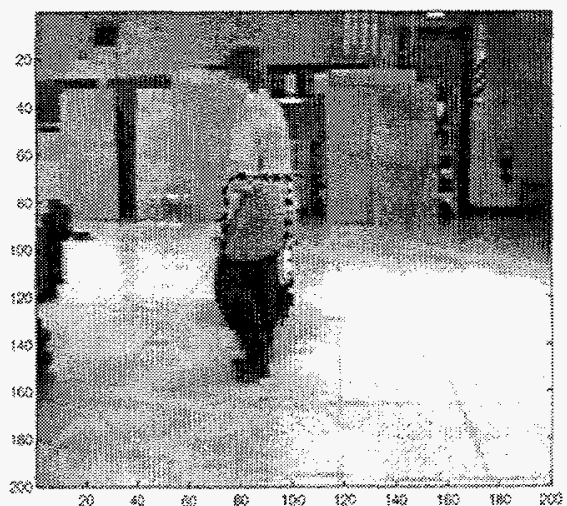

(d)

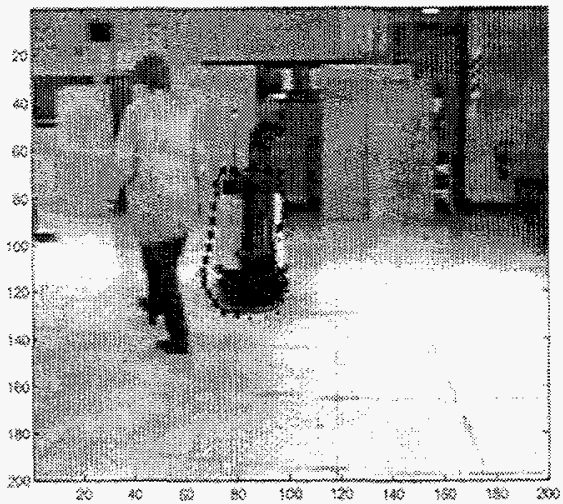

(g)

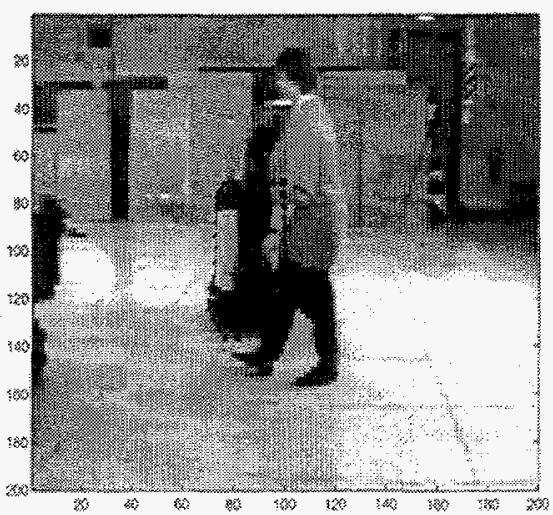

(b)

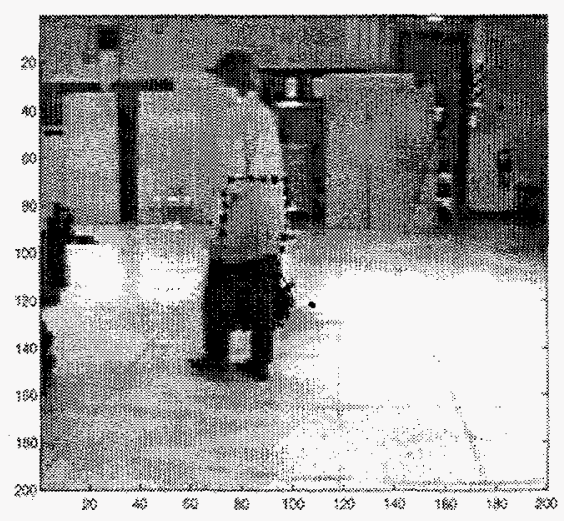

(e)

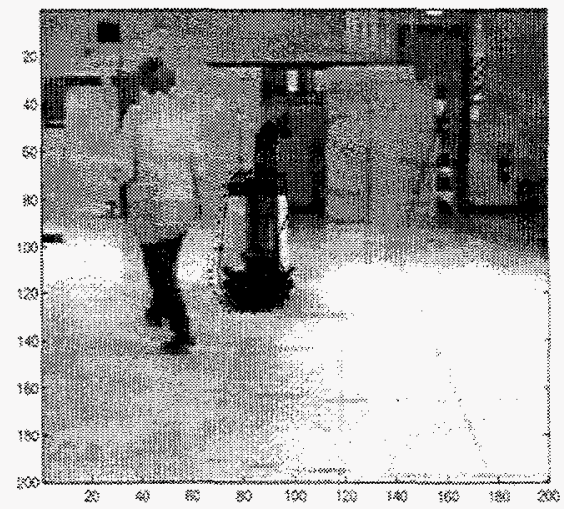

(h)

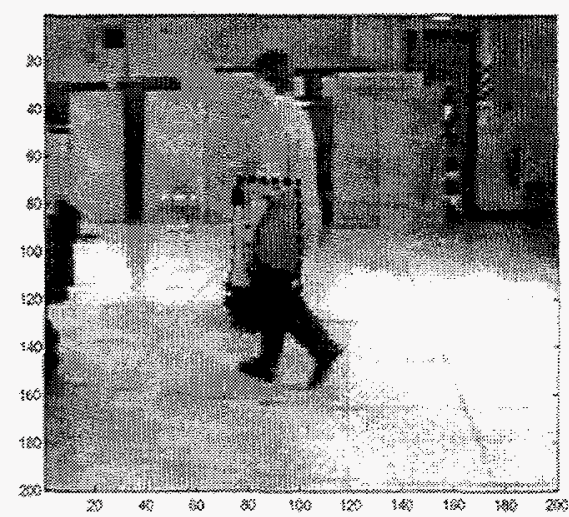

(c)

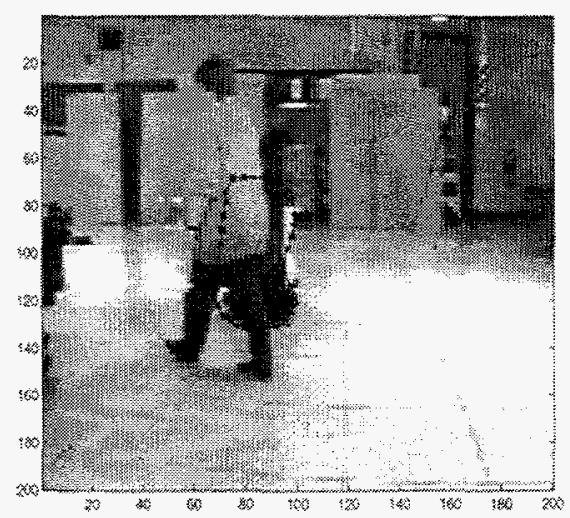

(f)

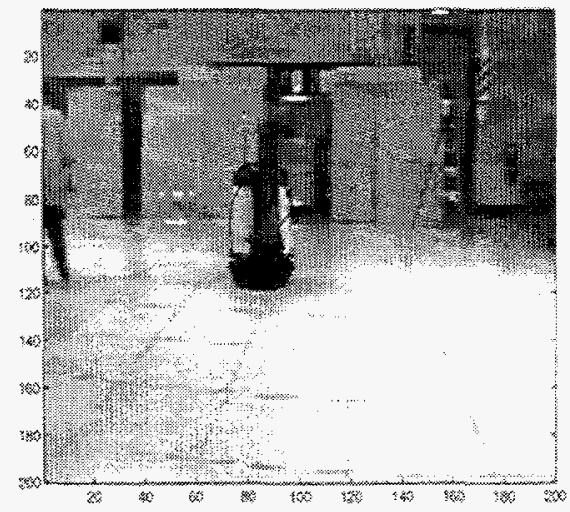

(i)

Figure 5.1: Tracking results of the mobile robot with the Batch Mode model and with the structured velocity (3.15)-(3.17). 


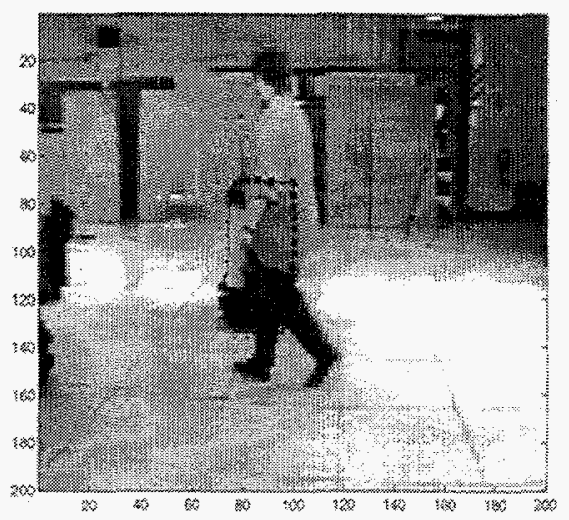

(a)

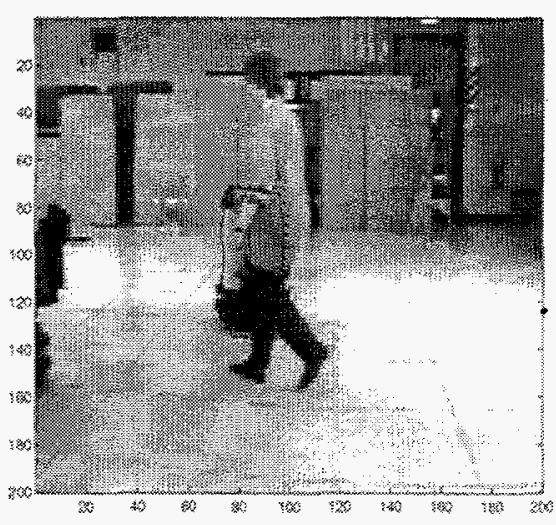

(c)

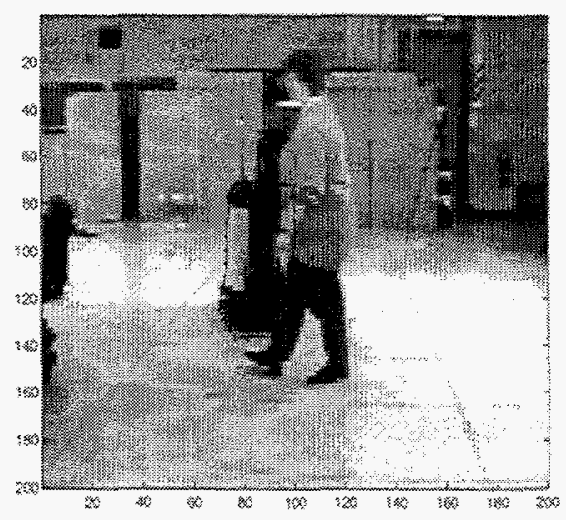

(e)

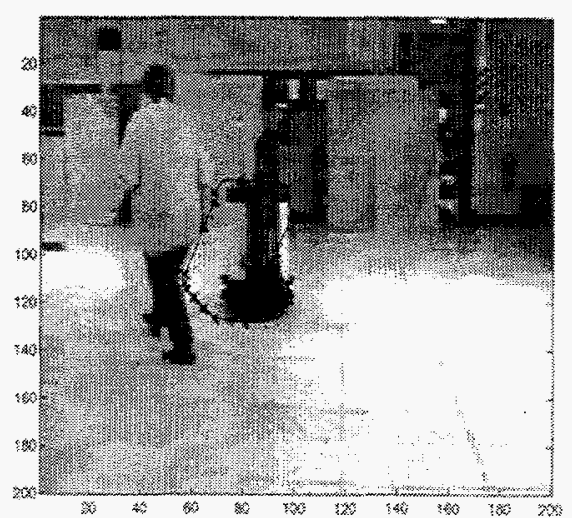

(b)

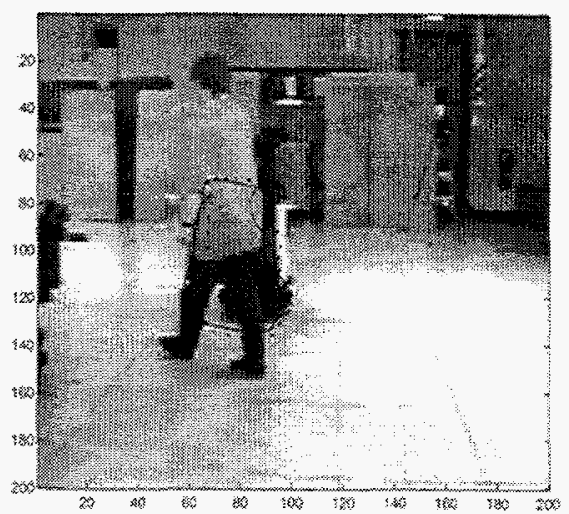

(d)

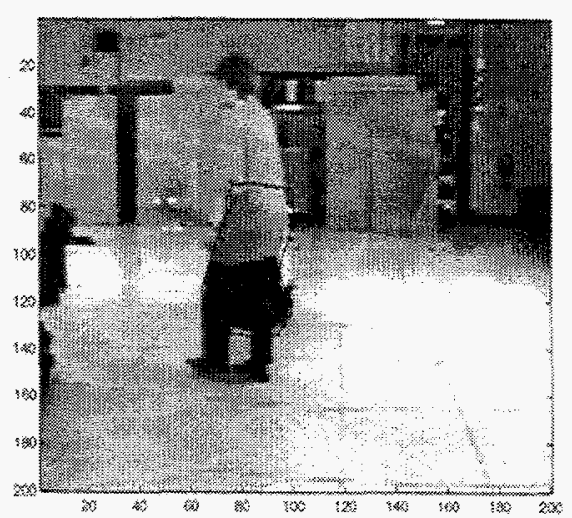

(f)

Figure 5.2 Tracking results of the mobile robot: (a)-(b). Batch Mode model with detection threshold $\gamma_{i}=4$, (c)-(d). Batch Mode model with no detection threshold, (e)-(f) The Kalman Snake model [19]. 


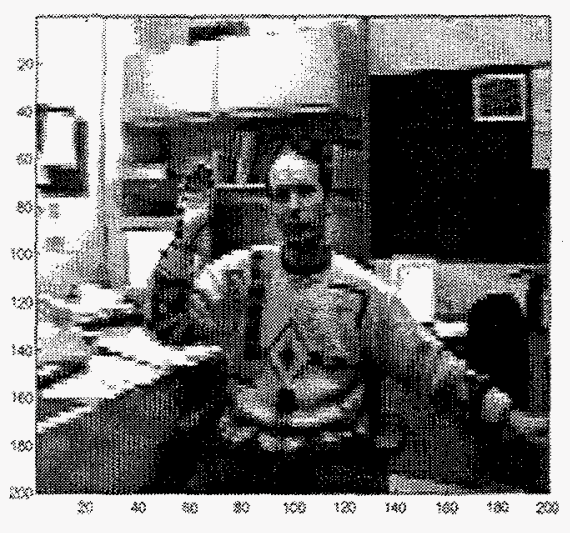

(a)

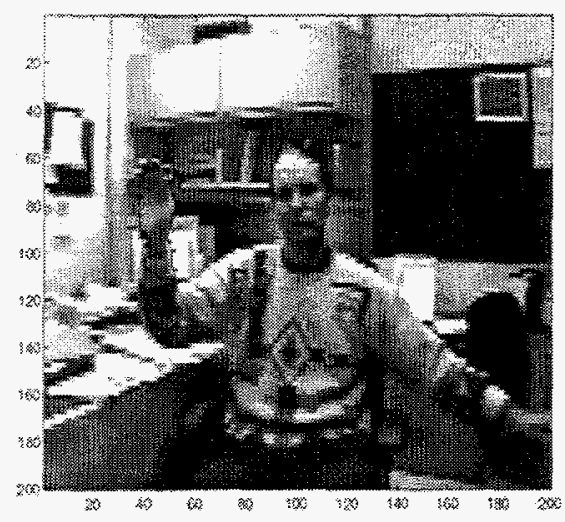

(d)

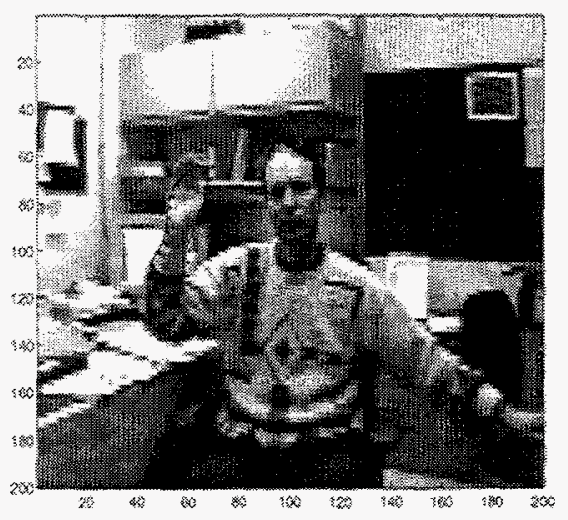

(f)

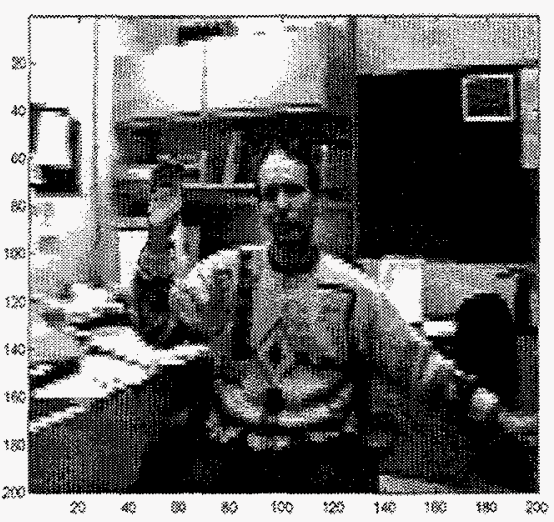

(b)

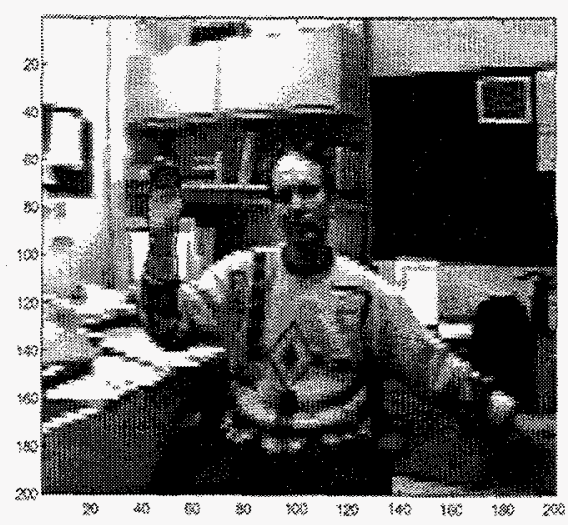

(c)

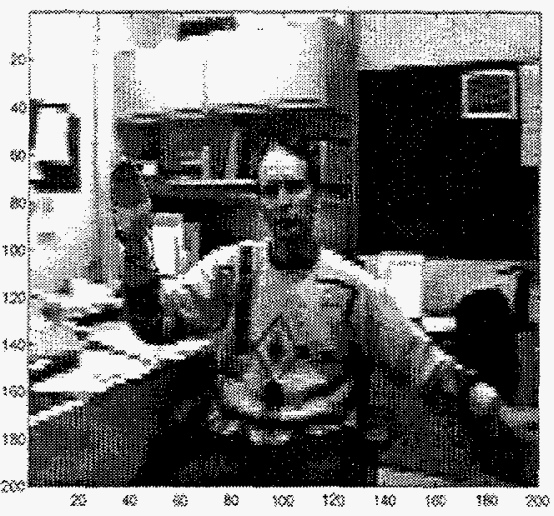

(e)

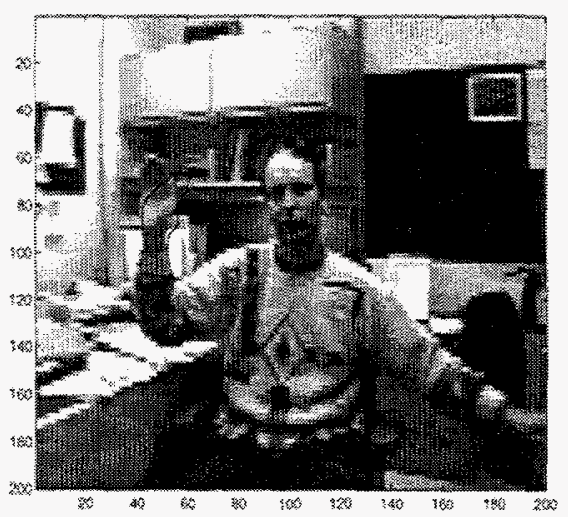

(g)

Figure 5.3: Tracking results of the waving hand: (a)-(e). Real Time model with detection threshold $\gamma_{i}=1,(\mathrm{f})-(\mathrm{g})$. The Kalman Snake model [19] 
of object velocity are space dependent, a model based image velocity was introduced. This model allows for velocity and position prediction in the presence of occlusions. In our case, however, it is limited to shallow objects. The new model was tested on a real data, resulting in good tracking performance and robustness to occlusions and image clutter. This result could be further improved by incorporating higher level models of shape within the snake and velocity models. These problems are the subject of current research.

\section{Acknowledgments}

This research was performed at the Center for Engineering Systems Advanced Research, Oak Ridge National Laboratory. Funding provided by the Engineering Research Program of the Office of Basic Energy Sciences under contract DE-AC05-96OR22464 with Lockheed Martin Energy Research, Corp. The author thanks Dr. Jacob Barhen, head of CESAR at ORNL.

\section{References}

[1] G. Adiv, Determining three-dimensional motion and structure from optical flow generated by several moving objects, IEEE Trans. Pattern Anal. and Machine Intell. PAMI 7(4), 1985, pp. 384-401.

[2] J.K. Aggarwal, Q. Cai, W. Liao and B. Sabata, Articulated and elastic non-rigid motion: A review, in Proc. of IEEE Computer Society Workshop on Motion of Non-Rigid and Articulated Objects, pp. 16-22, Austin, TX, 1994.

[3] K.J. Astrom and B. Wittenmark, Adaptive Control, Addison-Wesley, 1995.

[4] I.J. Cox and S.L. Hingorani, An Efficient Implementation of Reid's Multiple Hypothesis Tracking Algorithm and Its Evaluation for the Purpose of Visual Tracking, IEEE Trans. Pattern Anal. and Machine Intell. 18(2), 1996, pp. 138-150.

[5] Y. Bar-Shalom and T.E. Fortmann, Tracking and Data Association, Academic Press Inc., Orlando, Florida, 1988. 
[6] R. Curwen and A. Blake, Dynamic contours: Real-time active splines, in Active Vision (A. Blake and A. Yuille Eds.), pp. 39-58, MIT Press, 1992.

[7] M.P. Dubuisson, S. Lakshmanan-and A.K. Jain, Vehicle segmentation and classification using deformable templates, IEEE Trans. Pattern Anal. and Machine Intel. 18(3), 1996, pp. 293-308.

[8] M.S. Grewal and A.P. Andrew, Kalman Filtering: Theory and Practice, Prentice Hall Inc., Upper Saddle River, New Jersey, 1993.

[9] B.K.P. Horn and B.G. Schunk, Determining optical flow, Artificial Intelligence 17, 1981, pp. 185-204.

[10] M. Kass, A. Witkin and D. Terzopoulos, Snakes: Active contour models, Int. J. Computer Vision 1(4), 1987, pp. 321-331.

[11] F. Leymarie and M.D. Levine, Tracking deformable objects in the plane using an active contour models, IEEE Trans. Pattern Anal. and Machine Intell. 15(6), 1993, pp. 61.7-634.

[12] M.R. Luettgen, W.C. Karl and A.S. Willsky, Efficient multiscale regularization with applications to the computation of optical flow, IEEE Trans. Image Processing 3(1), 1994, pp. 41-64.

[13] R. Malladi, J.A. Sethian and B.C. Vemuri, Shape modeling with front propagation: A level set approach, IEEE Trans. Pattern Anal. and Machine Intell. 17(2), 1995, pp. $158-175$.

[14] D. Metaxas and D. Terzopoulos, Shape and nonrigid motion estimation through physicsbased sythesis, IEEE Trans. Pattern Anal. and Machine Intell. 15(6), 1993, pp. 580-591.

[15] N. Peterfreund, The Velocity Snake, IEEE Nonrigid and Articulated Motion Workshop, Virgin Islands, 1997.

[16] N. Peterfreund, The Velocity Snake: Deformable Contour for Tracking in Combined Spatio-Velocity Space, Submitted CVIU, 1997.

[17] R.N.P. Rao, Robust Kalman Filters for Prediction, Recognition and Learning, Technical Rep. 645, CS Dep. Univ. of Rochester, 1996.

[18] S. Rowe and A. Blake, Statistical mosaics for tracking, Image and Vision Computing 14(8), 1996, pp. 549-564. 
[19] D. Terzopoulos and R. Szeliski, Tracking with kalman snakes, in Active Vision (A. Blake and A. Yuille Eds.), pp. 3-20, MIT Press, 1992. 


\section{INTERNAL DISTRIBUTION}

1-5. J. Barhen

6. C. W. Glover

7. W. C. Grimmell

8. H. E. Knee

9. E. M. Oblow

10. C. E. Oliver

11-15. L. E. Parker

16-20. N. Peterfreund

21. F. G. Pin

22. V. Protopopescu
23. N. S. Rao

24. D. B. Reister

25-29. R. F. Sincovec

30. M. S. Smith

31. E. C. Uberbacher

32. M. A. Unseren

33-37. CSMD Reports Office

38. Laboratory Records - RC

39. Document Reference Section

40. Central Research Library

41. ORNL Patent Office

\section{EXTERNAL DISTRIBUTION}

42-43. Office of Scientific and Technical Information, P.O. Box 62, Oak Ridge, TN 37831.

44. Dr. Fred Aminzadeh, UNOCAL, 14141 SW Freeway, Suite 301-225, Sugarland, TX 77478.

45. Dr. John Baillieul, Aerospace \& Mechanical Engineering Department, Boston University, 110 Cummington Street, Boston, MA 02215.

46. Dr. John Blair, JBX Technologies, 25 Moore Road, Wayland, MA 01778.

47. Dr. Roger W. Brockett, Harvard University, Piece Hall, 29 Oxford Street, Cambridge, MA 02138.

48. Dr. Oscar P. Manley, 13212 Wye Oak Drive, Gaithersburg, MD 20878.

49. Dr. Robert E. Price, ER-15, Office of Basic Energy Sciences, Department of Energy, Washington, DC 20585.

50. Dr. Charles R. Weisbin, Robotics and Mars Exploration Technology Program, Jet Propulsion Laboratory, 4800 Oak Grove Drive, Pasadena, CA 91109. 